\title{
Inductive Power Link for a Wireless Cortical Implant with Biocompatible Packaging
}

\author{
Kanber Mithat Silay, Catherine Dehollain, Michel Declercq \\ Institute of Electrical Engineering, RFIC Research Group \\ Ecole Polytechnique Fédérale de Lausanne (EPFL) \\ CH-1015, Lausanne, Switzerland \\ e-mail: kanbermithat.silay@epfl.ch
}

\begin{abstract}
This article presents an inductive power link for a cortical implant. The link includes a Class-E power amplifier, an inductive link, a matching network, and a rectifier. The coils of the inductive link are designed and optimized for a distance of $10 \mathrm{~mm}$ (scalp thickness). The power amplifier is designed in order to allow closed loop power control by controlling the supply voltage. A new packaging topology is proposed in order to position the implant in the skull, without occupying much area, but still obtaining short distance between the remote powering coils. The package is fabricated using biocompatible materials such as PDMS and Parylene-C, and it includes the secondary coil, the matching network, and the rectifier. The power efficiency of the link is characterized for a wide range of load power (1$20 \mathrm{~mW}$ ) and found to be $8.1 \%$ for nominal load of $10 \mathrm{~mW}$. The matching network improves the power efficiency on the whole range, compared to the link without the matching network.
\end{abstract}

\section{INTRODUCTION}

With the recent developments in the microelectronics and MEMS technologies, it is possible to realize biomedical implants, which can be used for several different applications such as detection of blood glucose level, restoration of vision (retinal implants), regulation of heart beat (pacemakers), etc. Another application is to use a cortical implant for in vivo recording of the neural activity in the brain, in order to facilitate brain-machine interfaces [1], [2].

As in almost all types of biomedical implants, supplying power to the cortical implants is a challenge. It is possible to use direct transcutaneous wires or rechargeable batteries to power the implant. However, using wires may cause infections; whereas, batteries have limited recharge cycles and surgical operations may be necessary to replace them at the end of their lifetime. At last but not least, it is possible to supply power to the implants remotely.

Inductive links are the most commonly used technique for remote powering of biomedical implants [3]-[6]. External reader located outside the body transforms the electrical energy supplied from an external battery to a magnetic field, and then, the implant harvests energy from this magnetic field, generating a power supply for the active devices in the implant. As the external reader is operated from the battery, the power transfer should be as efficient as possible, in order to use a small and light-weight battery for patient mobility and comfort.

A typical remote powering link consists of four main parts: (1) power amplifier, (2) inductive link, (3) rectifier, and (4) voltage regulator. With respect to power efficiency, the bottleneck of the remote powering link is generally at the inductive link because the coupling factor between the power coils is usually very small. Therefore, the coils should be designed properly to obtain high power efficiency.

As shown in previous studies, it is possible to improve the power efficiency of the inductive link by properly designing the geometry of the coils [5]-[7]. Nevertheless, the efficiency is not only a function of the geometry of the coils but is also a function of the distance between the coils. In order to improve the power efficiency, this distance should also be kept as small as possible. The minimum distance between the two coils can be as high as $20 \mathrm{~mm}$ for cortical implants, due to the thicknesses of scalp and skull tissues [2], [8]. This distance can be decreased by changing the design of the implant packaging [9]. However, the electrode array in [9] is fixed to the skull, which may cause damage to the brain, if the head is subject to a physical impact.

This study presents the design of an inductive power link with a biocompatible package for a wireless cortical implant. First, the functions of the building blocks of the link are explained. Then, a new packaging topology for cortical implants is proposed, which decreases the distance between the power transfer coils to achieve high efficiency without increasing the surface area occupied by the implant. Finally, the simulation and measurement results for the inductive power link are presented to verify the operation of the proposed link.

\section{INDUCTIVE POWER LINK}

Fig. 1 shows the proposed remote powering link for a wireless cortical implant. The link is composed of a Class-E type power amplifier, an inductive link, a matching network, a rectifier, and a regulator. The power amplifier and the external part of the inductive link are located at the external reader, which is positioned outside the body; whereas, the implanted part of the inductive link, the matching network, the rectifier, and the regulator are located at the cortical implant inside the body.

\section{A. Inductive link}

The inductive link consists of a series resonance circuit at the external reader $\left(L_{1}\right.$ and $\left.C_{1}\right)$ and a parallel resonance tank at the implant $\left(L_{2}\right.$ and $\left.C_{2}\right)$. The geometries of the coils are 


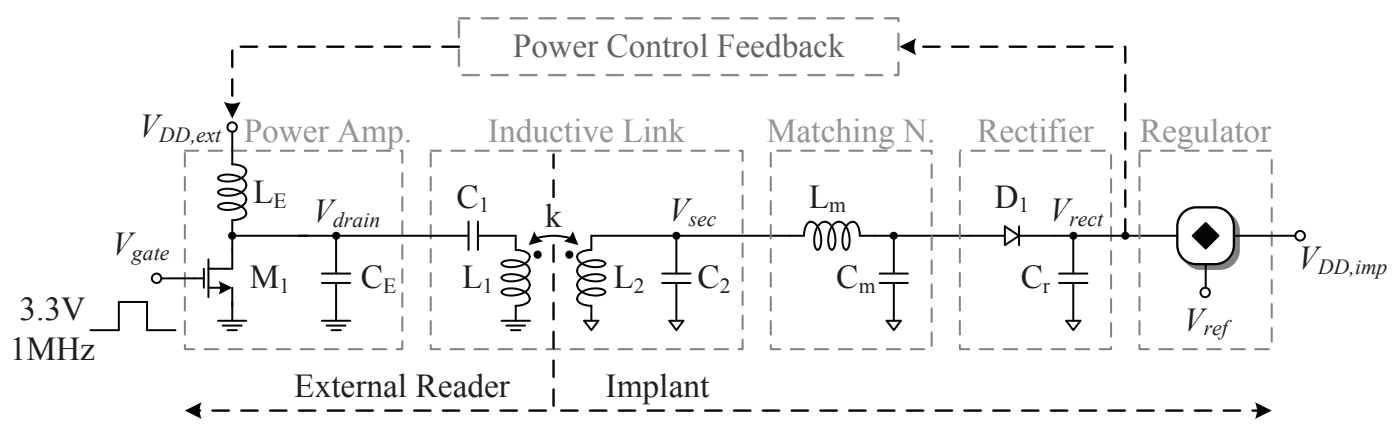

Fig. 1. Proposed remote powering link for a wireless cortical implant.

optimized by using a modified version of the optimization procedure presented in [6].

The operation frequency is chosen to be $1 \mathrm{MHz}$ in order to decrease absorption at the tissues [10]. Moreover, the distance between the reader and implant coils is chosen to be $10 \mathrm{~mm}$ to improve the power transfer efficiency (see Sec. III).

\section{B. Class-E power amplifier}

A Class-E type power amplifier is chosen for this application as the drain efficiency of this amplifier is usually very high [11]. When switching transistor $\left(M_{1}\right)$ in Fig. 1 is turned on, a flux is generated on the RF-choke inductor $\left(L_{E}\right)$. When the switch is turned off, the energy stored in the inductor will be transferred to the load of the amplifier. As the current through and voltage across the transistor are never high simultaneously, the power consumption on the transistor is very low. Therefore, the drain efficiency of the amplifier can be increased significantly, by careful adjusting of the load network of the amplifier.

A load network is necessary for Class-E amplifiers in order to remove harmonics generated from switching of the transistor $M_{1}$. This network is inherently present for the inductive power link as the primary coil $\left(L_{1}\right)$ and the capacitor $\left(C_{1}\right)$ acts as a bandpass filter at the operation frequency. Therefore, no additional component is required in order to filter the harmonics of the drain voltage. Moreover, the implant side resonance tank will further reject the harmonics as it is also a bandpass network around the operation frequency.

\section{Rectifier}

A simple half-wave rectifier topology is used in this study in order to rectify the ac signal generated at the implanted coil $\left(V_{s e c}\right)$. The matching network between the inductive link and the rectifier transforms the input impedance of the rectifier at a certain load to the optimum load resistance of the inductive link [12]. This method improves the power efficiency of the overall link around the specified power consumption.

The output of the rectifier should always be larger than a certain potential, in order to satisfy the operation of the voltage regulator following the rectifier. Therefore, it is essential to keep track of the rectifier output voltage $\left(V_{\text {rect }}\right)$, to ensure that the voltage regulator generates the desired supply voltage for the implant $\left(V_{D D, i m p}\right)$. This can be achieved by using a power control feedback network from the output of the rectifier to the power supply of the amplifier outside. The feedback information can be added to the recorded neural data at the implant and sent through the uplink, which is used for transferring information from the implant to the external reader. The power control feedback structure is currently under study and is not included in this article.

\section{Voltage regulator}

The voltage regulator is connected to the output of the rectifier to suppress the ripples and generate a clean supply voltage. The regulator topology will be based on the architecture proposed in [13] and is not presented in this study.

\section{PACKAGING}

Packaging is another challenge in the design of biomedical implants. The package should be biocompatible to prevent toxic and/or injurious effects to the patient, and also be hermetically sealed to separate the active parts from the fluidic environment in the body. Moreover, the package should be compact and small, in order not to occupy large surface area in the body.

A recent study proposes a compact assembly concept for a neural interface using Utah Microelectrode Arrays (MEA) [2]. The assembly concept includes all parts of the implant, including MEA, readout integrated circuit (IC), power receiving coil, etc. Although the implant occupies compact space, this type of assembly should be located under the skull, which causes the implanted coil to be far from the external reader coil. The distance between the two coils can be as large as $20 \mathrm{~mm}$, the total thicknesses of the scalp and the skull tissues [8].

Another conceptual cortical implant is proposed in [9]. In this type of packaging assembly, the implant is fixed in a cavity on the cranial bone. This allows the implanted power receiving coil (downlink coil) to be placed just under the scalp. Therefore, the distance between the external reader and the implant coils can be decreased by the thickness of the skull; hence, increasing the power efficiency. Moreover, with this type of packaging, another coil can be placed vertically, in order to send the recorded information to the outside (uplink coil). The cross-coupling between the downlink coil and the 


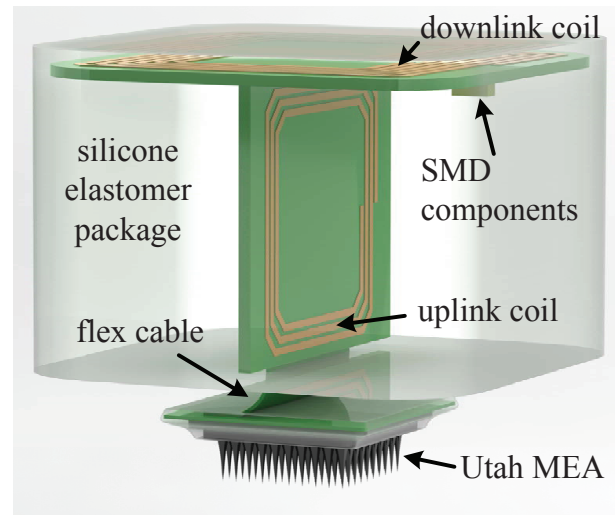

(a)

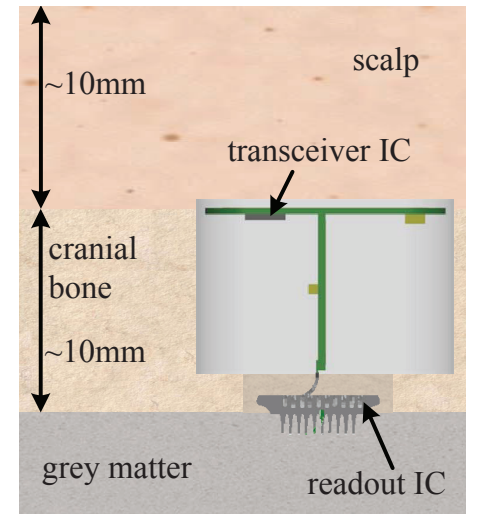

(b)

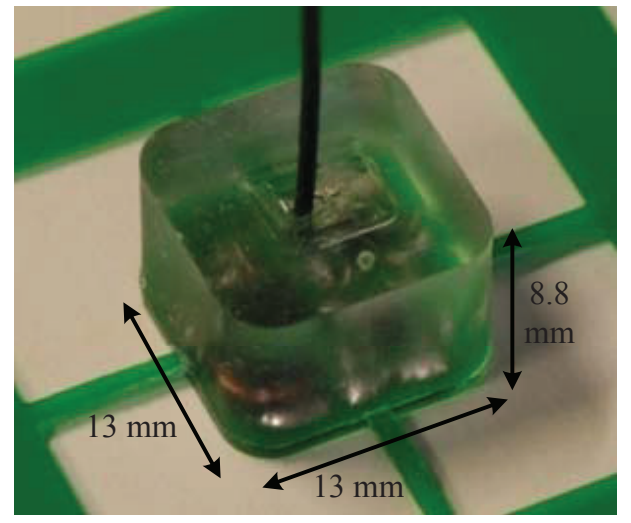

(c)

Fig. 2. Proposed packaging for a wireless cortical implant (a) Components of the package, (b) Position of the package inside the cranial bone, and (c) Photograph of the fabricated package with remote powering link.

uplink coil is small, as the coils are orthogonal to each other [9]. Finally, this type of packaging allows distributing the heat dissipation to a larger volume; hence, allowing more power consumption in the implant without damaging the surrounding tissues [14].

The disadvantage of the packaging concept in [9] is that the implant is a single piece block. When fixed to the skull, the electrodes may damage the brain, if the head is subject to a physical impact. To prevent this from happening, the electrodes should be mechanically decoupled from the skull. This can be achieved by using a flexible cable to connect the fixed part with the electrode array.

Fig. 2 shows the proposed packaging for a wireless cortical implant. The implant package has two parts: the readout part and the transceiver part. The transceiver part of the implant, which includes the coils and the transceiver IC, is fixed in a cavity on the cranial bone (see Fig. 2(b)). On the other hand, the readout part, which includes the readout IC and the electrodes, is connected to the transceiver part with a flexible cable. This flexible cable is used for transferring power and information between the two parts and it decouples the movable readout part from the fixed transceiver part.

For this type of packaging concept, as the implanted coil is separated from the external reader coil by only the thickness of the scalp, which is around $10 \mathrm{~mm}$, the coupling factor between the coils can be increased, resulting higher power efficiency.

The transceiver part of the package in Fig. 2(a) is fabricated by using a biocompatible silicone elastomer (Sylgard 184 PDMS). The fabricated package is coated conformally with $2 \mu \mathrm{m}$ Parylene $\mathrm{C}$ to improve its biocompatibility. Fig. 2(c) shows the photograph of the fabricated package with remote powering link. The transceiver part measures $13 \mathrm{~mm} \times 13 \mathrm{~mm}$ $\mathrm{x} 8.8 \mathrm{~mm}$ and it includes the implant side of the inductive link, the matching network, and the rectifier.

\section{Characterization Results}

Table I presents the fixed system parameters used during optimization of the inductive link. The optimal load resistance
TABLE I

Fixed System Parameters for the Remote Powering Link

\begin{tabular}{|l|c|l|}
\hline Parameter & Value & Explanation \\
\hline \hline$f_{0}$ & $1 \mathrm{MHz}$ & Operation frequency \\
$R_{s r c}$ & $0.5 \Omega$ & Modeled amplifier resistance \\
$h$ & $25 \mu \mathrm{m}$ & Conductor thickness \\
$s_{\min }$ & $100 \mu \mathrm{m}$ & Minimum conductor spacing \\
$w_{\min }$ & $100 \mu \mathrm{m}$ & Minimum conductor width \\
od $d_{i, \max }$ & $10 \mathrm{~mm}$ & Max. outer dimension of implanted coil \\
$d$ & $10 \mathrm{~mm}$ & Distance between the coils \\
\hline
\end{tabular}

during optimization is chosen to be $27 \Omega$, considering the compromise between the power efficiency and the susceptibility of the coils to parasitics [12]. The designed coils are fabricated on printed circuit boards.

Fig. 3 shows the simulated and measured power efficiency of the inductive link vs. load resistance of the inductive link. As expected, the optimum load for this design is around $27 \Omega$. The maximum measured inductive link efficiency is $14.85 \%$ at $1 \mathrm{MHz}$.

The gate of the switching transistor of the Class-E power amplifier is driven from a function generator with a square wave of $3.3 \mathrm{~V}$ peak-to-peak and $1.65 \mathrm{~V} \mathrm{DC}$ offset at $1 \mathrm{MHz}$. In order to improve the drain efficiency, the Class-E power amplifier is tuned to have the desired drain voltage waveform, by changing the tuning capacitor $\left(C_{E}\right.$ in Fig. 1). The drain efficiency of the power amplifier is measured to be around $85 \%$. The losses in the amplifier are due to the finite quality factor of the RF-choke inductor and non-zero voltage drop across the switching transistor when it is turned on. The losses at the gate of the amplifier are not considered in this study.

The rectifier output voltage should not be less than $1.5 \mathrm{~V}$, for proper operation of the voltage regulator. Therefore, the desired rectifier output voltage is chosen to be $1.6 \mathrm{~V}$, leaving some margin for the ripples. The input impedance of the rectifier is simulated at its nominal load of $10 \mathrm{~mW}$ at $1.6 \mathrm{~V}$, 


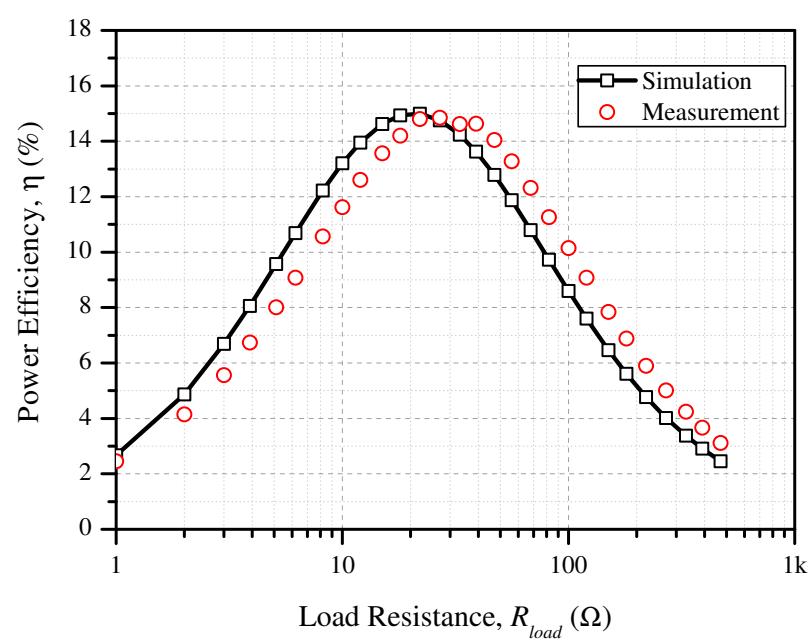

Fig. 3. Simulated and measured power efficiency of the inductive link vs. load resistance of the inductive link.

which approximately corresponds to a $253 \Omega$ load resistor. Then, the matching network is designed to transform the simulated input impedance of the rectifier at the operation frequency of $1 \mathrm{MHz}$, to the optimum load of the inductive link, which was set as $27 \Omega$ for this study.

In order to see the dependence of the DC voltage at the output of the rectifier with respect to the DC supply voltage of the power amplifier, the amplifier supply voltage $\left(V_{D D, e x t}\right)$ is swept for a certain load, and the rectifier output voltage is monitored $\left(V_{\text {rect }}\right)$. Fig. 4 shows the rectifier output voltage vs. Class-E supply voltage for $253 \Omega$ load resistance at the output of the rectifier. As seen from this figure, the relation of $V_{\text {rect }}$ with respect to $V_{D D, e x t}$ is quite linear, due to the linearity of the Class-E power amplifier. The rectifier also does not affect the linearity much, as the drop across the diode is fairly constant for different currents drawn from the load. The linearity of the rectifier output voltage with respect to power amplifier supply voltage simplifies the aforementioned power control feedback.

Fig. 5 displays the measured power efficiency of the overall remote powering link with and without the matching network. The losses at the voltage regulator and the gate of the power amplifier are not included in these results. The load power in Fig. 5 is defined as the power delivered to a resistive load at the output of the rectifier for $V_{\text {rect }}=1.6 \mathrm{~V}$. As seen from this figure, the use of the matching network between the inductive link and the rectifier increases the overall power efficiency, as the inductive link is operated near its optimum load condition. For nominal load of $10 \mathrm{~mW}$, the power efficiency of the remote powering link is increased to $8.1 \%$ by using matching network, compared to $4.5 \%$ efficiency measured without the matching network. The maximum measured power efficiency of the link with the matching network is $8.3 \%$ at $7 \mathrm{~mW}$, which is 2.4 times the power efficiency without the matching network. Similarly, the efficiency is improved by 5.8 times for $1 \mathrm{~mW}$ by using the matching network topology.

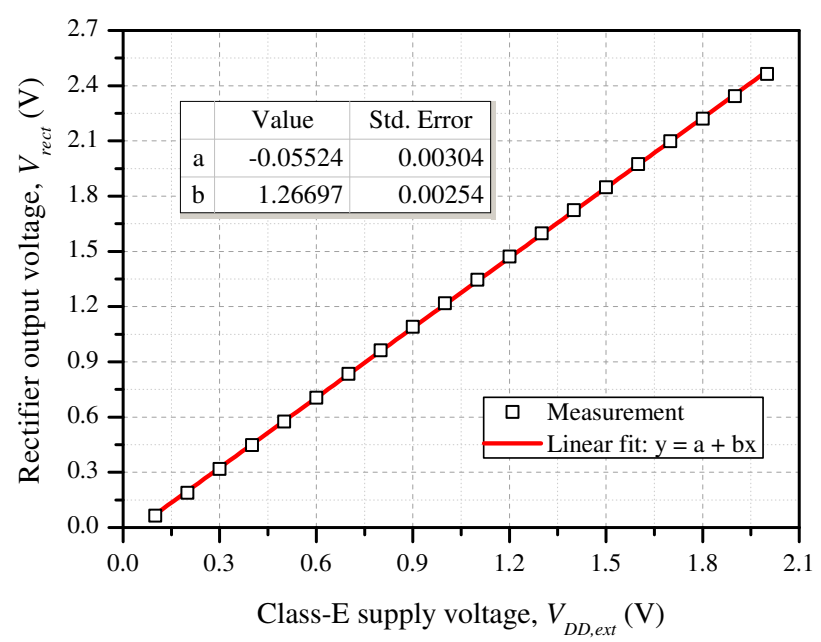

Fig. 4. Rectifier output voltage vs. Class-E supply voltage $\left(R_{\text {load }}=253 \Omega\right)$.

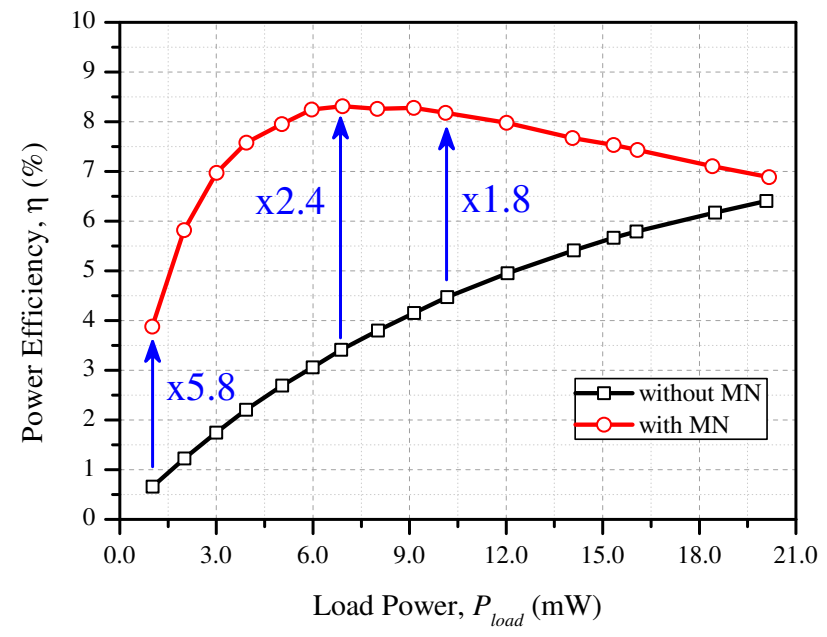

Fig. 5. Measured power efficiency of the overall remote powering link with and without the matching network (regulator and gate losses not included).

Fig. 6 shows the measured waveforms of the remote powering link for $10 \mathrm{~mW}$ delivered from the rectifier at $1.6 \mathrm{~V}$ $\left(R_{\text {load }}=253 \Omega\right)$. As seen from the drain waveform of the power amplifier, the voltage across the transistor is close to zero, when the gate signal is high ( $M_{1}$ is on). When the gate signal is low ( $M_{1}$ is off), there is no current flowing through the drain of the transistor. Therefore, the power consumption at the transistor is very small, resulting in a highly efficient power amplifier.

\section{CONCLUSiON}

In this study, an inductive remote powering link for a wireless cortical implant is presented. The remote powering link consists of a Class-E power amplifier, an inductive link, a matching network, and a rectifier. The operation of each block of the link is summarized and methods for increasing the power efficiency of the overall link are explained. 


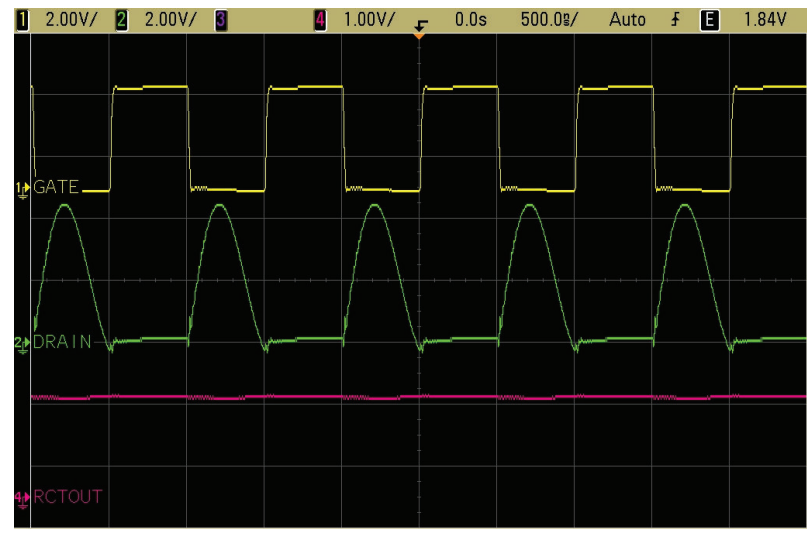

Fig. 6. Measured waveforms of the remote powering link for $10 \mathrm{~mW}$ delivered from the rectifier at $1.6 \mathrm{~V}$ (yellow $=V_{\text {gate }}$, green $=V_{\text {drain }}$, pink $\left.=V_{\text {rect }}\right)$.

A new type of biocompatible packaging concept for cortical implants is introduced, which allows the power receiving coil of the implant to be placed to a closer distance of the external reader coil. As the coils can be placed closer, the power efficiency of the inductive link can be increased.

The fixed part of the proposed package, which is used for generating the power supply and communicating with outside, can be positioned inside a cavity on the skull. On the other hand, the movable part is placed on the brain for recording the neural activity. The two parts can be connected together with a flexible cable for transferring power and information. This allows the readout part to move with the brain, in order not to damage the neurons, if the head is subject to a physical impact. The transceiver part of the package, which will be fixed to the cranial bone, is fabricated by using a biocompatible silicone elastomer and covering it with Parylene C.

The performance of the remote powering link is characterized by measuring the power efficiency from the supply of the power amplifier to the output of the rectifier, excluding the gate losses at the amplifier. When a matching network is used between the inductive link and the rectifier, the power efficiency is measured to be $8.1 \%$ for $10 \mathrm{~mW}$ power delivered from the rectifier, compared to $4.5 \%$ efficiency without the matching network. The maximum measured efficiency is $8.3 \%$ with the matching network, 2.4 times the efficiency without the matching network. Moreover, the efficiency is increased to 5.8 times the efficiency of the link without matching, for $1 \mathrm{~mW}$ power consumption at the rectifier load. The matching network improves the power efficiency between $1 \mathrm{~mW}$ and $20 \mathrm{~mW}$, the desired power consumption range for the implant.

The dependence of the rectifier output voltage with respect to power amplifier supply voltage is also characterized. As expected, the relation is linear, which simplifies the design of a power control loop.

The realization of the remote powering link with the regulator is under progress. The effect of the tissues on the power efficiency should also be investigated and is currently under study.

\section{ACKNOWLEDGMENT}

The NEURO-IC project is supported by the Swiss National Funding (SNF). Authors would like to thank H.C. Tekin and Prof. M. Gijs from EPFL Microsystems Laboratories for their helps in packaging. Authors also would like to thank Dr. S. Ayoz and Y. Temiz for their feedbacks.

\section{REFERENCES}

[1] K. D. Wise, D. J. Anderson, J. F. Hetke, D. R. Kipke, and K. Najafi, "Wireless implantable microsystems: High-density electronic interfaces to the nervous system," Proc. IEEE, vol. 92, no. 1, pp. 76-97, 2004.

[2] R. R. Harrison, P. T. Watkins, R. J. Kier, R. O. Lovejoy, D. J. Black, B. Greger, and F. Solzbacher, "A low-power integrated circuit for a wireless 100-electrode neural recording system," IEEE J. Solid-State Circuits, vol. 42, no. 1, pp. 123-133, 2007.

[3] S. Atluri and M. Ghovanloo, "A wideband power-efficient inductive wireless link for implantable microelectronic devices using multiple carriers," in Proc. IEEE ISCAS'06, 2006, pp. 1131-1134.

[4] C. Sauer, M. Stanacevic, G. Cauwenberghs, and N. Thakor, "Power harvesting and telemetry in CMOS for implanted devices," IEEE Trans. Circuits Syst. I, vol. 52, no. 12, pp. 2605-2613, 2005.

[5] C. M. Zierhofer and E. S. Hochmair, "Geometric approach for coupling enhancement of magnetically coupled coils," IEEE Trans. Biomed. Eng., vol. 43, no. 7, pp. 708-714, 1996.

[6] K. M. Silay, C. Dehollain, and M. Declercq, "Improvement of power efficiency of inductive links for implantable devices," in Proc. PRIME'08 Conf., 2008, pp. 229-232.

[7] R. R. Harrison, "Designing efficient inductive power links for implantable devices," in Proc. IEEE ISCAS'07, 2007, pp. 2080-2083.

[8] National Library of Medicine, USA. (2007) The Visible Human Project. [Online]. Available: http://www.nlm.nih.gov/research/visible/

[9] K. M. Silay, C. Dehollain, and M. Declercq, "Orthogonally oriented coils for minimization of cross-coupling in cortical implants," in Proc. IEEE BioCAS'08, 2008, pp. 109-112.

[10] P. Vaillancourt, A. Djemouai, J. F. Harvey, and M. Sawan, "EM radiation behavior upon biological tissues in a radio-frequency power transfer link for a cortical visual implant," in Proc. IEEE EMBC'97, 1997, pp. 24992502 .

[11] N. O. Sokal and A. D. Sokal, "Class E-A new class of high-efficiency tuned single-ended switching power amplifiers," IEEE J. Solid-State Circuits, vol. 10, no. 3, pp. 168-176, 1975.

[12] K. M. Silay, D. Dondi, L. Larcher, M. Declercq, L. Benini, Y. Leblebici, and C. Dehollain, "Load optimization of an inductive power link for remote powering of biomedical implants," in Proc. IEEE ISCAS'09, 2009, pp. 533-536.

[13] V. Majidzadeh, A. Schmid, and Y. Leblebici, "A fully on-chip LDO voltage regulator for remotely powered cortical implants," in Proc. ESSCIRC'09, 2009, pp. 424-427.

[14] K. M. Silay, C. Dehollain, and M. Declercq, "Numerical analysis of temperature elevation in the head due to power dissipation in a cortical implant," in Proc. IEEE EMBC'08, 2008, pp. 951-956. 